\title{
EMC aware design and testing of intermodulation distortion under multiple co-located sources illumination
}

\author{
Alessandro Biondi, Luigi Vallozzi, Frederick Declercq, Hendrik Rogier and Daniël De Zutter
}

\begin{abstract}
Current electromagnetic immunity tests mainly rely on single-frequency sources. However, the evolution of electronic systems leads to miniaturization and low-cost solutions, in which filters are omitted in front of active non-linear components, also for efficiency reasons. As a result, intermodulation products may leak into the band of operation. We propose a comprehensive strategy consisting of design and test methodologies to evaluate in-the-band leakage of out-of-band undesired components, using multiple-tone excitation and relying on an anechoic chamber as test facility. The aim of this paper is to demonstrate that an anechoic chamber together with a dual-source network analyzer represents an optimal facility to investigate signal integrity issues due to leakage of intermodulation products.
\end{abstract}

\section{Index Terms}

Intermodulation products, electromagnetic immunity, signal integrity, non-linear devices

\section{INTRODUCTION}

$\mathbf{I}$ $\mathrm{N}$ the last years, a great interest has emerged in the development of compact, low-cost, low-power, reconfigurable microwave systems. This evolution led to miniaturized, low-cost solutions, in which filters in front of active devices [1] may be omitted. For instance, in software defined radio (SDR), a very broadband signal is received by the antenna and directly amplified, before being converted to the digital domain, where further processing, including filtering, takes place [2]. Non-linearities in these devices combined with the lack of shielding and filtering may compromise performance, signal integrity and electromagnetic (EM) compatibility [3]-[5] due to in-the-band leakage of out-of-band undesired signals interfering with the desired signal. Indeed, when two or more signals are present at the input of a non-linear component, the signal at the output of that component includes other frequency components in addition to the wanted signals due to intermodulation (IM) distortion. This issue in particular arises when several sources illuminate devices under test (DUTs), where none of the sources will individually compromise signal integrity (SI) or EM immunity (EMI) but the total effect of these sources is such as to potentially cause SI and EM compatibility (EMC) problems [6].

Therefore, in contrast to existing established EMC tests, it is important to develop and understand approaches to test electronic systems under multiple-sources illumination, in particular when IM products occur that might potentially disturb the DUT and cause SI/EMI issues. Existing EMC tests rely on single frequency sources, and thereby do not always provide a realistic transmission scenario [6], [7].

Hence, in this paper, we present a comprehensive experimental strategy to model and test RF devices under multiple frequency sources illumination, in the presence of out-of-band frequency components leaking into the band of interest. Specifically, the novel contributions are: (i) a new EMC aware computer-aided design strategy is outlined providing guidelines to account for in-the-band leakage of undesired out-of-band signals due to IM distortion. It is demonstrated how the current use of standard parameters describing non-linearities, such as $P_{1 \mathrm{~dB}}, \mathrm{OIP}_{2}$ and $\mathrm{OIP}_{3}$ often fail to capture leakage effects; (ii) novel precompliance tests using a dual-source Vector Network Analyzer are proposed at the subsystem/device level to quickly identify and correct for IM distortion during the design; (iii) new EMC tests are defined to characterize in-the-band leakage of a DUT, using an anechoic chamber and multiple co-located interfering sources transmitted along the main beam of the illuminating antenna, as such representing the worst-case transmission scenario. Test procedures are outlined where the DUT is tested at different radiated power levels and at varying distances.To the authors' knowledge, this is the first paper presenting a real comprehensive multi-frequency modeling and test strategy in the EMC context.

The organization of the paper is as follows. First, the different mechanisms that lead to in-the band leakage of undesired signals via IM distortion are discussed in the next section. This allows us to define modelling and test procedures during the design, precompliance as well as compliance testing phases. Sections III and IV discuss the practical implementation of this strategy for a representative circuit, consisting of an antenna that picks up the disturbing signals in a frequency-selective way combined with a low-noise amplifier (LNA) that creates intermodulation distortion (IMD) due to its non-linearity. Specifically, in the design and precompliance testing phase, as discussed in Section III, the focus is on the non-linear device, tested and 
modeled in a $50 \Omega$ environment. Section IV focusses on testing the complete DUT for EMC compliance. Section V summarizes the conclusions of the paper.

\section{Accounting for IM Distortion in EMC Aware Design And Testing}

In this section we put forward a strategy to evaluate the behavior of a DUT (Fig. 1) under multiple-sources' illumination. In particular, we must account for all different causes of IM products that might give rise to in-the-band leakage.

\section{A. Causes of IM distortion}

Electronic devices are susceptible to different kinds of disturbances depending on their nature. In order for a component device or system to be susceptible to multi-frequency signals it must consist of a concatenation of a susceptible linear device, and a non-linear component creating IM products. Specifically, it is the absence of a filter in between the susceptible device and the non-linear component in Fig. 1 that allows undesired out-of-band signals to leak into the frequency band of operation. To provide a clear view to the EMC aware design and test engineer, we first present the different scenarios resulting in IM distortion. Each cause of IM products, leading to potential in-the-band leakage of unwanted signals due to IM distortion, corresponds to a specific multi-frequency transmission scenario that will be recreated in the design, precompliance and compliance testing phase by means of modeling and experiments. Fig. 2 shows the case in which a transmitter illuminates the DUT with a single frequency component (tone) at $f_{0}$, and a higher-order (the second-order in the figure) harmonic falls into the band of interest as unwanted signal (scenario 1). Once this occurs, the unwanted signal can no longer be removed by filtering. Therefore, it is important to test whether the power of this in-the-band disturbance remains small enough compared to the desired signal, in order not to affect the EMC performance of the DUT.

[Fig. 1 about here.]

[Fig. 2 about here.]

The second scenario, represented in Fig. 3, depicts the case of two tones, $f_{1}$ and $f_{2}$, a desired one $f_{1}$ falling in the band, and an unwanted one $f_{2}$ falling out-of-band, radiated by the co-located sources illuminating the DUT ( two possible cases $f_{2}$ $<f_{1}$ or $f_{2}>f_{1}$ ). In this scenario the two frequency components result in an intermodulation product falling in the band of operation of the device. Again, the power of the in-the-band interference should be monitored to validate the EMC behavior of the DUT. The results reported later in Section IV only consider the case $f_{2}<f_{1}$ but similar results could be obtained for $f_{2}>f_{1}$. Note that also two undesired out-of-band signals generating relevant in-the-band interference should be considered in this scenario, although such a configuration is not illustrated in the sequel for reasons of conciseness.

[Fig. 3 about here.]

Finally, Fig. 4 depicts a third scenario in which a high power out-of-band transmitter (frequency $f_{2}$ ) is illuminating the DUT. Because of its high power, the LNA does no longer operate as expected. The LNA gets desensitized i.e. gain and linearity decrease, resulting in a desired signal that is less amplified and hence more difficult to detect. Also this scenario should be accounted for in EMC aware design and suitable tests must be established to ensure EM compatibility of the DUT.

At this point we should make two important remarks: First, from Fig. 4 it is seen that the undesired signal that desensitizes the amplifier may also result in important in-the-band IM products, as studied in scenario two. In such a case, the desensitization will further decrease the ratio of the power of the desired signal over the power of the IM distortion, as the wanted signal gets less amplified whereas the unwanted signal increases more than expected with rising signals levels. Second, a strong out-of-band signal may desensitize the DUT without producing IM products in the desired frequency band. This process may, however, make the DUT more sensitive to IM products produced by other out-of-band signals. This, rather complex, scenario is not further investigated for reasons of conciseness.

[Fig. 4 about here.]

\section{B. Design and precompliance/compliance testing strategies}

To be able to include IM distortion during the EMC aware design phase, the interfering multi-frequency signals will be applied as sources in a full-wave/circuit co-simulation, whereas during precompliance tests these simulated results are verified at the subsystem and module levels by performing direct multi-tone injection by means of an Agilent N5242A PNA-X network analyzer. Testing all subsystems/modules separately provides a quick way for early diagnostics during design and precompliance testing, but it does not provide a complete picture, since input and/or output of the subsystem/module under test act as frequencydependent loads over the broad frequency range over which unwanted signals will impinge, and this is not taken into account when using a network analyzer, which tests the subsystem/module in a constant $50 \Omega$ environment.

[Fig. 5 about here.] 
Therefore in the final compliance tests the multi-frequency signals are transmitted by a standard gain horn letting them impinge in the far-field on the complete DUT. In Fig. 5 we propose the setup for such a multi-frequency test to be performed in an anechoic chamber. The transmission source in the experiment is a standard gain horn, connected to a highly-linear power amplifier, and finally connected to an Agilent N5242A PNA-X network analyzer used as multi-frequency generator. The choice of the power amplifier is crucial as will be discussed in section IV. As we are dealing with non-linear phenomena, varying power levels must be considered. Instead of varying the power level we may make the distance between the transmitter and the DUT variable, where the lower bound of that distance must still respect the far-field condition. In the sequel, the in-the-band frequency range is defined by its lower $f_{L}$ and its upper frequency $f_{H}$. In our experiments we focus on the ISM-band extending from $2.4 \mathrm{GHz}$ up to $2.4835 \mathrm{GHz}$, but for the ease of the discussion we choose $f_{L}=2.4 \mathrm{GHz}$ to be the lower bound and $f_{H}=2.5 \mathrm{GHz}$ to be the upper bound of the frequency band of interest.

In the remaining part of this paper, by way of example, we will illustrate the aforementioned strategy to model and test the three different IM distortion scenarios by considering a simple susceptible passive component, combined with a simple active component that causes IMD. The DUT (Fig. 6) we consider is an active textile antenna [8] where the susceptible component is the passive antenna, and the active component is a low-noise amplifier (LNA). This example is of practical importance since in many such designs the presence of a frequency selective filter placed between the antenna and the LNA is omitted to reduce cost and to obtain a more compact and more efficient device. The LNA is directly fed by the antenna signal through a via without any matching network [8], choosing as antenna impedance the optimal impedance $(35.05-j 17.644) \Omega$ for minimum noise figure [8]. No filtering operation is provided by intermediate stages in the DUT.

[Fig. 6 about here.]

In the next section, we first consider the EMC aware design and precompliance testing phase, which focusses on the behavior of the LNA as a stand-alone subsystem/module. The final compliance testing of the complete DUT, being the active antenna, is discussed in Section IV.

\section{EMC Aware Design And Precompliance Testing of the LNA}

In contrast to the compliance testing phase, during EMC aware design and precompliance testing, the design engineers have the possibility to model, measure and manipulate the generation and propagation of IM products in isolated subsystems and modules. Hence, including IM distortion in the EMC aware design and precompliance testing cycles offers a cheap and efficient way to avoid in-the-band leakage of undesired signals in the final product. Let us now discuss our strategy by focussing on the LNA module of the active antenna. Models and measurements in this section are based on an LNA that is terminated by $50 \Omega$ at both input and output ports.

Low-noise amplifiers are traditionally characterized by their OIP3, OIP2 and $P_{1 d B}$ levels. Table I compares the results obtained through simulations with Agilent's Advanced Design System (ADS) to the values measured with the Agilent N5242A PNA-X Network Analyzer at $2.45 \mathrm{GHz}$. A good agreement is obtained between simulated and measured data. However, the interpretation of these numbers requires awareness of the specific modeling/measurement setup that led to each of these results. We note that $P_{1 d B}$ was obtained by injecting a single tone at $2.45 \mathrm{GHz}$, whereas two in-the-band tones spaced by $10 \mathrm{MHz}$ and centered around $2.45 \mathrm{GHz}$ were injected to find OIP3. Yet, to study OIP2, a single tone, now at $1.225 \mathrm{GHz}$, is again considered, observing the circuit's response at $2.45 \mathrm{GHz}$. In a real situation, the input power levels will never be that high as to e.g. reach the third order interception point. Furthermore, the actual non-linear circuit will exhibit a frequency-dependent behavior, given that all components possess frequency-dependent characteristics, specifically when considering the effect of unwanted out-of-band components that may be present in a very wide frequency band, much wider than intended by the design engineer. Hence, in EMC aware design and testing, we must carefully analyze the problem for different tone spacings and power levels.

Therefore, to characterize the effect of out-of-band signals, the Spurious Free Dynamic Range (SFDR) is put forward as a suitable measure. In literature [9], [10] several definitions of the SFDR can be found. For our purposes, we define SFDR as the ratio between the desired component and the highest spurious one leaking into the band(s) of operation, as schematically shown in Fig.7. In EMC aware design an upper limit for this SFDR should be set and modelled/tested for different combinations of desired and/or unwanted signals. In this paper, by way of example, we will consider a spurious component to be acceptable as long as the SFDR is higher than $40 \mathrm{~dB}$. However, it must be kept in mind that the optimum threshold is strictly applicationdependent.

We first discuss the relevancy in terms of EMC aware design of the modelling/measurement data that led to the results of Table I. These data are the gain at $2.45 \mathrm{GHz}$, the SFDR for two tones centered at $2.45 \mathrm{GHz}\left(f_{1}=2.445 \mathrm{GHz}\right.$ and $f_{2}=2.455$ $\mathrm{GHz}$ ) and the power of the second-order harmonic modelled/measured for varying power levels, as shown in Fig. 8. A good agreement is found between simulated and measured gain and for the power of the second-order harmonic but less so for the SFDR. The SFDR results refer to the ratio between the in-the-band tone and the highest in-the-band intermodulation product. The measured SFDR value stays above $40 \mathrm{~dB}$ for an input power lower than $-3 \mathrm{dBm}$. For the sake of completeness, $P_{N F}$ 
(Power Noise Floor) and $P_{M D S}$ (Power Minimum Detectable Signal) were also measured by means of the Agilent N5242A PNA-X Network Analyzer and turn out to be equal to $-90 \mathrm{dBm}$ and $-52 \mathrm{dBm}$, respectively.

Although the gain plot demonstrates desensitization at higher input levels, this result is not relevant for our purposes as it is only caused by in-the-band signals. Hence, extra filtering will not affect this curve. In the same way, the SFDR result, produced by two in-the-band components, will not change when introducing filters before the non-linear component. Hence, for an EMC engineer studying in-the band leakage of undesired components, only data related to the second-order harmonic offers relevant information. To that end an SFDR must be introduced comparing the power of the second-order harmonic leaking into the band to the typical power levels of the desired signals encountered in the application.

Therefore, we now focus on two tones' excitations that typically result in important in-the-band leakage when omitting a filter before the non-linear component. The first regions that require our attention are the boundaries of the frequency band(s) of operation. Fig. 9 shows the output spectrum of the LNA when excited with two tones: in the top figure these tones are located at $f_{2}=2.395 \mathrm{GHz}$ and $f_{1}=2.405 \mathrm{GHz}$, (i.e. a spacing of $10 \mathrm{MHz}$ centered at the lower end of the ISM band), whereas in the bottom figure the two tones are centered around the upper bound of the band, at $f_{1}=2.495 \mathrm{GHz}$ and $f_{2}=2.505 \mathrm{GHz}$. The values given in Fig. 9 are for an input power level of $P_{i n}=P_{1 d B}$, with $P_{1 d B}=-0.498 \mathrm{dBm}$ (see Table I). This high input power level was deliberately selected in order to obtain the worst case results. The results of Fig. 9 clearly show the existence of unwanted in-the-band tones. However, the SFDR remains above $40 \mathrm{~dB}(+43.54 \mathrm{~dB})$.

[TABLE 1 about here.]

[Fig. 7 about here.]

[Fig. 8 about here.]

[Fig. 9 about here.]

[Fig. 10 about here.]

Next, the power level of the out-of-band tone is kept at $P_{1 d B}$ but the in-the-band tone power level is decreased to -10 $\mathrm{dBm}$. This is considered to be relevant for the test phase because in a real transmission context it is very likely that an out-of-band component can be stronger in power than the in-the-band one. The obtained results are shown in Fig. 10. We observe a difference of $59.35 \mathrm{~dB}$ between the in-the-band tone and the highest spurious component falling within the band. Hence, the SFDR is again acceptable. Besides these example configurations, other two-tone excitations, even two out-of-band signals, may result in relevant in-the-band leakage. However, filtering thanks to the frequency dependency of the susceptible linear component may reduce the power of these leaking IM products, as discussed in the next section.

\section{Compliance Testing of the Active Antenna}

[Fig. 11 about here.]

Let us now turn to the compliance testing phase of the DUT (Fig. 11), in which all relevant scenarios leading to in-the-band leakage should be verified. To generate a strong enough incident signal, two highly linear power amplifiers are cascaded: the highly linear power amplifier contained in the Agilent N5242A PNA-X Network Analyzer and a Mini-Circuits ZRL-3500 power amplifier providing a maximum gain of $G_{P A}=21 \mathrm{~dB}$. The choice for that type of amplifier is not arbitrary. Indeed, we must make sure that its linearity is superior to that of the LNA which is part of our DUT. If this would not be the case, our test set-up would indeed not make sense. This makes clear that the choice for a particular additional amplifier in the measurement chain depends on the specific DUT one is interested in. The Mini-Circuits $Z R L-3500$ power amplifier (further refered to as MC amplifier) has an OIP3 of $+45 \mathrm{dBm}$. The maximum allowed input power is specified to be $+10 \mathrm{dBm}$. However, when using the MC amplifier in a cascade with the PNA-X amplifier, careful experiments have revealed that this input power must be further restricted in order to drive the second amplification stage without generating IMD, distortion which could perturb the measurement results of the DUT. Several tests were performed to characterize the amplifier cascade in terms of linearity, by driving the input of the MC amplifier with a variable power (emitted by the PNA-X) ranging from -20 to $0 \mathrm{dBm}$. Up to $0 \mathrm{dBm}$ no significant IMD turns up and hence this $0 \mathrm{dBm}$ will be the highest input power level at the MC amplifier used for the DUT measurements. To define the relevant power range of desired and unwanted signals incident from the standard gain horn onto the DUT we rely on the Equivalent Isotropically Radiated Power (EIRP) [11], being the amount of power that the standard gain horn emits to produce the peak power density in the direction of maximum antenna gain. Referring to Fig. 11, the EIRP is given by

$$
E I R P[\mathrm{dBm}]=P_{\mathrm{in}[P N A-X]}[\mathrm{dBm}]+G_{P A}[\mathrm{~dB}]+G_{H O R N}[\mathrm{dBi}]-L_{[\text {cables }]}[\mathrm{dB}]-M_{t}[\mathrm{~dB}],
$$

with $G_{H O R N}$ the gain of the standard gain horn (Scientific Atlanta SGH-1.1 or 1.7, depending on the desired excitation frequency ), $L_{\text {cables }}$ the cable losses and with $M_{t}$ representing the transmission mismatch. The relevant numbers at $2.45 \mathrm{GHz}$ are summarized in Table II. We must also take into account that the maximum allowed EIRP that may be radiated by emitters 
in the $2.45 \mathrm{GHz}$ ISM band is limited in Europe to $+20 \mathrm{dBm}$ by ETSI EN 300328 and by the US FCC Rules, Part 15.247, to $+36 \mathrm{dBm}$.

\section{[TABLE 2 about here.]}

[Fig. 12 about here.]

The EIRP is related to the signal at the output of the active textile antenna by means of

$$
P_{\text {out }}[\mathrm{dBm}]=E I R P[\mathrm{dBm}]-P L[\mathrm{~dB}]+G_{D U T}[\mathrm{~dB}]-M_{r}[\mathrm{~dB}]
$$

with $P L[\mathrm{~dB}]$ the path loss, depending on the distance $d$ between SGA and DUT (Fig. 11), $M_{r}$ the mismatch factor at the receiver and $G_{D U T}[\mathrm{~dB}]=G_{A n t D U T} \times G_{L N A}$ the gain of the complete DUT. $G_{D U T}$ is the product of $G_{A n t D U T}$, the gain of the passive susceptible device, and $G_{L N A}$, the transducer gain of the non-linear component with the antenna as its source. Although (2) relates to the linear behavior of the DUT, the generation of IM products is directly related to the output level of the DUT. Hence, the IM distortion will be influenced by the frequency selectivity of the path loss and, more importantly, by $G_{D U T}$. Therefore, it is important to first characterize the gain of the DUT as a function of frequency through a separate S-parameter measurement, by

$$
G_{D U T}[\mathrm{~dB}]=S_{21 \text { meas }}[\mathrm{dB}]-M_{r}[\mathrm{~dB}]-M_{t}[\mathrm{~dB}]+G_{P A}[\mathrm{~dB}]-G_{H O R N}[\mathrm{dBi}]+P L[\mathrm{~dB}]
$$

$S_{21 \text { meas }}$ represents the measured forward transmission coefficient. The top graph of Fig. 12 shows the measured $G_{D U T}$ as a function of frequency. To characterize the effects of frequency-selective gain, we define the Transmission Attenuation (TA) as the absolute value of the difference between the maximum value of $G_{D U T}$ and $G_{D U T}$ as a function of frequency. The resulting TA is shown in the bottom graph of Fig. 12. For these measurements we took the distance $d$ to be $10 \lambda_{1}$, with $\lambda_{1}$ the wavelength at $2.45 \mathrm{GHz}$. We observe that the DUT already provides $3 \mathrm{~dB}$ of attenuation at $2.385 \mathrm{GHz}$ and $2.5 \mathrm{GHz}, 10 \mathrm{~dB}$ at $2.2 \mathrm{GHz}$ and $2.6 \mathrm{GHz}$, and $15 \mathrm{~dB}$ at $1.7 \mathrm{GHz}$ and $2.7 \mathrm{GHz}$. As proposed in Section II, we now investigate the behaviour of the DUT using the three proposed scenarios.

1) First Scenario (Fig. 2): In our measurement, $f_{0}=1.225 \mathrm{GHz}$ was used and while sweeping the input power we observe the occurrence of an in-the-band harmonic at $2 f_{0}=2.45 \mathrm{GHz}$. The test was performed for different distances between the standard gain horn and the DUT. Fig. 13 shows the second-order harmonic's power over a range of distances from 8 down to $5 \lambda_{2}$ (where $\lambda_{2}$ is the free-space wavelength at $1.225 \mathrm{GHz}$ ). The power of the second-order harmonic remains smaller than $-52.47 \mathrm{dBm}$ with $d=5 \lambda_{2}$ for an EIRP of $+20 \mathrm{dBm}$. To reach an SFDR of 40dB i.e. our proposed threshold limit, the desired signal should reach at least $-12.47 \mathrm{dBm}$ for the DUT to pass this EMC test.

[Fig. 13 about here.]

2) Second Scenario (Fig. 3): The two tones radiated by the horn in our experiment are $f_{2}=2.395 \mathrm{GHz}$ and $f_{1}=2.405$ $\mathrm{GHz}$, with, as for the LNA testing, a spacing of $10 \mathrm{MHz}$. They have the same power. The EIRP used for the experiments was varied from a minimum of $+10 \mathrm{dBm}$ up to $+37 \mathrm{dBm}$. The distance between horn and DUT was varied from 9 to $12 \lambda_{1}$ (free-space wavelength at $2.45 \mathrm{GHz}$ ). The results shown in Fig. 14 and in Fig. 15 refer to a distance of $9 \lambda_{1}$ and EIRP levels of $+27,+30,+32$ and $+34 \mathrm{dBm}$. For an EIRP of $+20 \mathrm{dBm}$ the intermodulation products just reach the noise floor $(-90$ $\mathrm{dBm}$ ) and the level of the main tones is $-27.31 \mathrm{dBm}$. In Fig. 16, SFDR values are reported which rely on the measurements performed at different distances between the standard gain horn and the DUT. Considering an SFDR threshold of $40 \mathrm{~dB}$, and depending on the distance between the radiating element and the DUT, we can state that the SFDR stays above the threshold for EIRP values smaller than $32 \mathrm{dBm}$ and for a distance larger than $9 \lambda_{1}$.

[Fig. 14 about here.]

[Fig. 15 about here.]

[TABLE 3 about here.]

Similar results are obtained at the upper side of the frequency band and for other distances. Table III summarizes the power level of the largest in-the-band main tone $P_{\text {Tone, }}$ and of the strongest in-the-band third-order intermodulation product $P_{T o n e, 3}$ for various EIRP values, at a distance $d=9 \lambda_{1}$. It is seen that the SFDR drops below $40 \mathrm{~dB}$ for EIRP $\geq 32 \mathrm{dBm}$.

[Fig. 16 about here.]

3) Third Scenario (Fig. 4): Let us now focus on how desensitization affects the SFDR. As already mentioned, any strong undesired signal may reduce the amplification of the desired signal and thereby decrease the SFDR. In most cases, the most critical configuration will be encountered when a strong interfering signal is present near the boundaries of the frequency band of operation, simultaneously causing IM products and desensitization in the DUT. Therefore, again consider the experiment that resulted in Figs. 14, 15 and 16. At the higher EIRP levels we clearly observe that desensitization starts influencing the performance of the DUT. The power of the main tones no longer increases at the same rate as for the lower EIRP values while 
the intermodulation products grow faster. To characterize this phenomenon an important parameter describing the behavior of the DUT under the variation of EIRP is introduced: the Tone Power Variation $T P V_{n}$, defined as

$$
T P V_{n}=\left.\frac{P_{\text {Tone }, n}}{E I R P}\right|_{P L=\text { constant }} .
$$

In Fig. 17, $T P V_{1}$ and $T P V_{3}$ are shown as a function of EIRP, for different distances between transmitter and DUT. In particular, by calculating this variation we can identify the power level at which the amplification starts decreasing, due to the high input power level, for each transmitter-DUT separation. When increasing the EIRP level we expect the power of the main tones to increase by the same quantity and the third-order intermodulation products to grow cubically until the LNA contained in DUT desensitizes due to saturation, exhibiting lower gain, resulting in a higher growth rate for the intermodulation products and in a lower growth for the main tones. This is clearly visible in Fig. 17: around $+30 \mathrm{dBm}$ of EIRP (for the closest distance between the test antenna and the DUT equal to $9 \lambda_{1}$ ) the LNA starts desensitizing.

[Fig. 17 about here.]

[Fig. 18 about here.]

[Fig. 19 about here.]

This effect will be even more pronounced when considering a dual-tone co-located transmitter illuminating the DUT, as in the second scenario, but now assuming the out-of-band tone to be much higher in power than the desired signal. In particular, here we choose this unwanted component to be $10 \mathrm{~dB}$ higher in power. $E I R P_{1}$ represents the power level applied to the out-of-band tone, while $E I R P_{2}$ represents the power level applied to the in-the-band component. In the top part of Fig. 18 we observe that the intermodulation products occur at the same level as the noise floor, similar to the case with equal power for the out-of-band and in-the-band component. In Fig. 19 we notice that by increasing the $E I R P_{1}$ to resp. $+32 \mathrm{dBm}$ and +34 $\mathrm{dBm}$, and $E I R P_{2}$ to resp. $+22 \mathrm{dBm}$ and $+24 \mathrm{dBm}$, the in-the-band intermodulation product rises to $-62.25 \mathrm{dBm}$ and -51.07 $\mathrm{dBm}$, respectively. We observe that the third-order intermodulation product grows faster than cubically due to the influence of the out-of-band component. This phenomenon tends to be more noticeable when increasing the EIRP level. Indeed, for $E I R P_{1}$ $=+32 \mathrm{dBm}$ we observe a difference of $2.39 \mathrm{~dB}$ between the expected in-the-band intermodulation product and the measured one. For the case $E I R P_{1}=+34 \mathrm{dBm}$ the difference equals $3.13 \mathrm{~dB}$. This effect quickly reduces the available SFDR. Indeed, for $E I R P_{1}=+32 \mathrm{dBm}$, the SFDR level equals $30.85 \mathrm{~dB}$, whereas when going to $E I R P_{1}=+34 \mathrm{dBm}$, the SFDR drops to $18.98 \mathrm{~dB}$

\section{CONCLUSions}

Current EMC testing procedures have most often focused on single-source/single-frequency illumination of the devices under test. In general there are no conventional test practices that involve multi-source/multi-frequency testing. Therefore, we have proposed a new modeling and experimental approach dealing with intermodulation distortion phenomena in a transmission context by means of multiple co-located sources. This new approach is suitable for any generic DUT composed of at least one active non-linear component and a passive susceptible antenna. An active textile antenna (consisting of an LNA and a passive antenna) was used as test bench to prove the effectiveness of the new approach with the $2.45 \mathrm{GHz}$ ISM-band as the frequency band of interest. By analyzing and measuring the components composing the DUT (in our case the LNA and the passive antenna part) and by subsequently measuring the entire DUT in several transmission scenarios using horn antennas in an anechoic chamber with a PNA-X network analyzer as multi-frequency source, it was shown how a better and more correct knowledge about leakage of undesired signals into the DUT's frequency band of operation when illuminating with a a single out-of-band frequency (scenario 1) or a combination of an in-the-band and an out-of-band frequency (scenario 2) can be obtained. To characterize the effect of harmonics of the out-of-band frequency turning up in the frequency band of interest, the SFDR was put forward as a suitable measure. Furthermore, the influence of strong transmitters, desensitizing the LNA, on the relative importance of intermodulation products in the DUT's output spectrum was carefully examined (scenario 3). As a measure for the desensitization, we introduced the Tone Power Variation parameter, i.e. the ratio of the power of a particular tone to the EIRP of the standard gain horn. For these measurements, an additional highly-linear pre-amplifier (in our case the Mini-Circuits ZRL-3500 power amplifier) was necessary. Concluding, it can be stated that quantitative testing of active DUT's with non-linear components illuminated by multiple co-located sources emitting both desired, in-the-band, and undesired, out-of-band, signals can be successfully carried out using an anechoic chamber, standard gain horns, a PNA-X network analyzer and a well-chosen linear preamplifier together with a set of clear measurement scenarios.

\section{REFERENCES}

[1] A. Rembovsky, A. Ashikhmin, V. Kozmin, S. Smolskiy, Radio Monitoring: Problems, Methods and Equipment, 1st ed. Springer, 2009.

[2] K. A. R. P. Cruz, N. B. Carvalho, "Designing and testing software designed radios," IEEE Microwave Magazine, pp. 83-94, June 2010.

[3] I. T. 61000-1-2, "Electromagnetic compatibility (EMC) - part1-2: general-methodology for the achievement of the functional safety of electrical and electronic equipment with regard to electromagnetic phenomena," February 2008. [Online]. Available: http://iec.ch 
[4] M. Mardiguian, "Combined effects of several simultaneous, EMI couplings," vol. 1, Washington, August 2000, pp. 181-184.

[5] J. Nitsch, N. Korovkin, E. Soloveyna, and H.-J. Scheibe, "Occurrence of low-frequency noises in electronic systems under action of two-tone high frequency electromagnetic excitation," vol. 2, Magdeburg, August 2005, pp. 618-621.

[6] Duffy, A., Orlandi, A. , Armstrong, K., "Preliminary study of a reverberation chamber method for multiple-source testing using intermodulation," IET Sci. Meas. Technol., vol. 4, pp. 21-27, 2010.

[7] Armstrong, K., "Functional safety requires much more than EMC testing," EMC-Europe, Sept. 2004, pp. 348-353.

[8] F. Declercq and H. Rogier, "Active integrated wearable textile antenna with optimized noise characteristics," IEEE Trans.Antennas Propag., vol. 58, no. 3, pp. 3050-3054, Sep. 2010.

[9] F. Span Application Note, "Spurious Free Dynamic Range." [Online]. Available: http://www.fiber-span.com

[10] E. Lawrence, RF and Microwave Circuit Design For Wireless Communications, 1st ed. Artech House, 1997.

[11] C. A. Balanis, Antenna Theory: Analysis and Design, 2nd ed. New York: John Wiley \& Sons, 1997. 


\section{LIST OF FIGURES}

A general DUT.

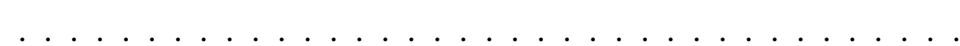

Propagation of harmonics in the output spectrum of the DUT (scenario 1). . . . . . . . . . . . . 10

Response of the DUT to an in-the-band tone $f_{1}$ and an out-of-band tone $f_{2}$ (scenario 2). . . . . . . . 11

Desensitization due to an out-of-band tone $f_{2}$ (scenario 3$) . \ldots \ldots \ldots$

Anechoic Chamber Setup. . . . . . . . . . . . . . . . . . . . . . . . 13

Passive antenna and LNA. . . . . . . . . . . . . . . . . . . . . . . . . . . . . . 14

Worst-case identification for the calculation of the Spurious Free Dynamic Range. . . . . . . . . . . . . . 15

8 Gain at $f_{0}=2.45 \mathrm{GHz}$, SFDR for $f_{1}=2.445 \mathrm{GHz}$ and $f_{2}=2.455 \mathrm{GHz}$, and second-order harmonic power for $f_{0}=1.225 \mathrm{GHz}$, simulated and measured. . . . . . . . . . . . . . . . . . . . . 16

9 Output spectrum with $P_{i n}=P_{1 d B}$ at the lower and at the upper side of the considered frequency band, for $\Delta f$ $=10 \mathrm{MHz}$ and excitation by two equal power tones $f_{1}$ and $f_{2} \ldots \ldots \ldots$

10 Measurement results as in Fig. 9 but now for two tones with a power difference of $10 \mathrm{dBm}$. . . . . . . . . 18

11 DUT measurement setup in the anechoic chamber. . . . . . . . . . . . . . . . . . . . 19

$12 G_{D U T}$ and $T A$ as a function of frequency, with $d=10 \lambda_{1} \ldots \ldots \ldots$

13 Second harmonic's power versus distance $d$ between the standard horn and the DUT. . . . . . . . . . . . . . . 21

14 Output spectrum for the excitation by two equal power tones in the proximity of the lower side of the frequency band with $\mathrm{EIRP}=+27 \mathrm{dBm}$ and $+30 \mathrm{dBm}$ respectively, using $d=9 \lambda_{1} \ldots \ldots$

15 Output spectrum for the excitation by two equal power tones in the proximity of the lower side of the frequency band with $\mathrm{EIRP}=+32 \mathrm{dBm}$ and $+34 \mathrm{dBm}$ respectively, using $d=9 \lambda_{1} \ldots \ldots . \ldots$

16 SFDR as a function of EIRP for two equal power tones and varying distances $\left(\lambda=\lambda_{1}\right)$. . . . . . . . . . 24

$17 T P V_{1}$ and $T P V_{3}$ as a function of EIRP and for variable distances $\lambda=\lambda_{1}$ between horn and DUT. . . . . . . .

18 Output spectrum for the excitation by two tones with different power in the proximity of the lower side of the frequency band and resp. for $E I R P_{1}=27 \mathrm{dBm}, E I R P_{2}=17 \mathrm{dBm}$ and for $E I R P_{1}=30 \mathrm{dBm}, E I R P_{2}=20$

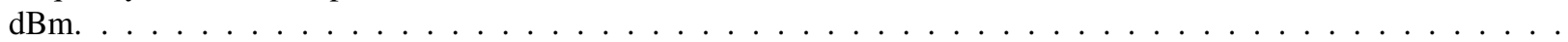

Output spectrum for the excitation by two tones with different power in the proximity of the lower side of the frequency band and resp. for $E I R P_{1}=32 \mathrm{dBm}, E I R P_{2}=22 \mathrm{dBm}$ and for $E I R P_{1}=34 \mathrm{dBm}, E I R P_{2}=24$

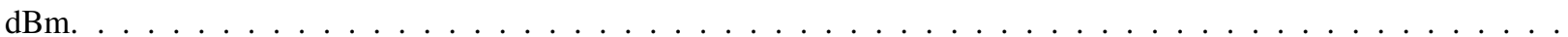




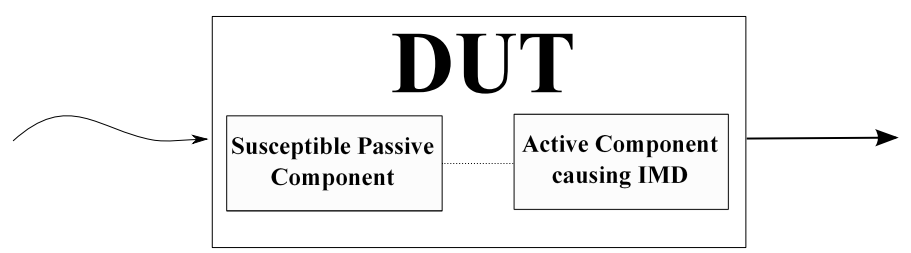

Fig. 1: A general DUT. 


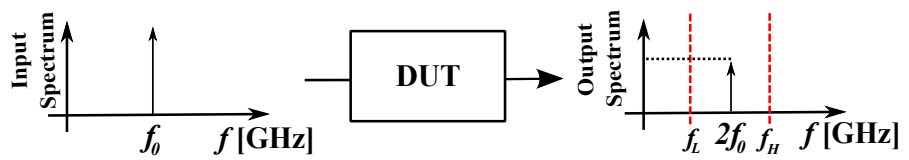

Fig. 2: Propagation of harmonics in the output spectrum of the DUT (scenario 1). 


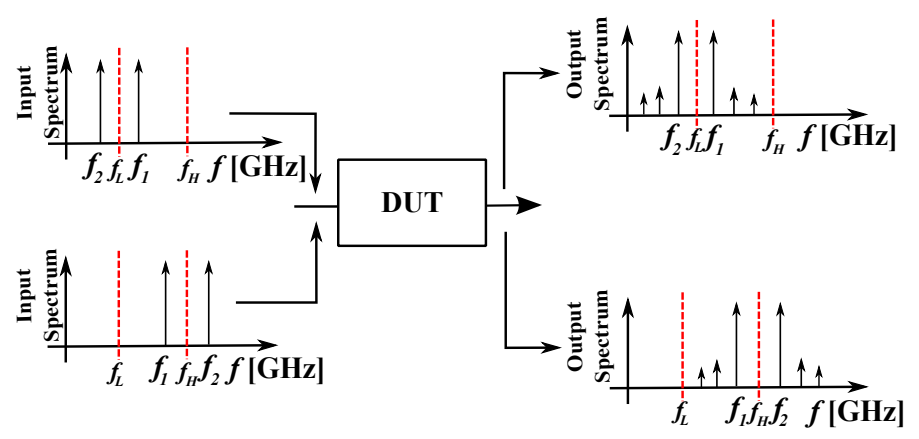

Fig. 3: Response of the DUT to an in-the-band tone $f_{1}$ and an out-of-band tone $f_{2}$ (scenario 2). 
Strong out-of-the-band

$$
\text { transmitter }
$$

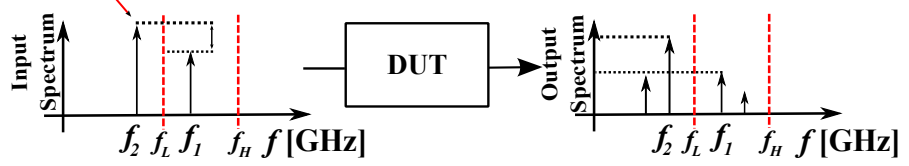

Fig. 4: Desensitization due to an out-of-band tone $f_{2}$ (scenario 3). 


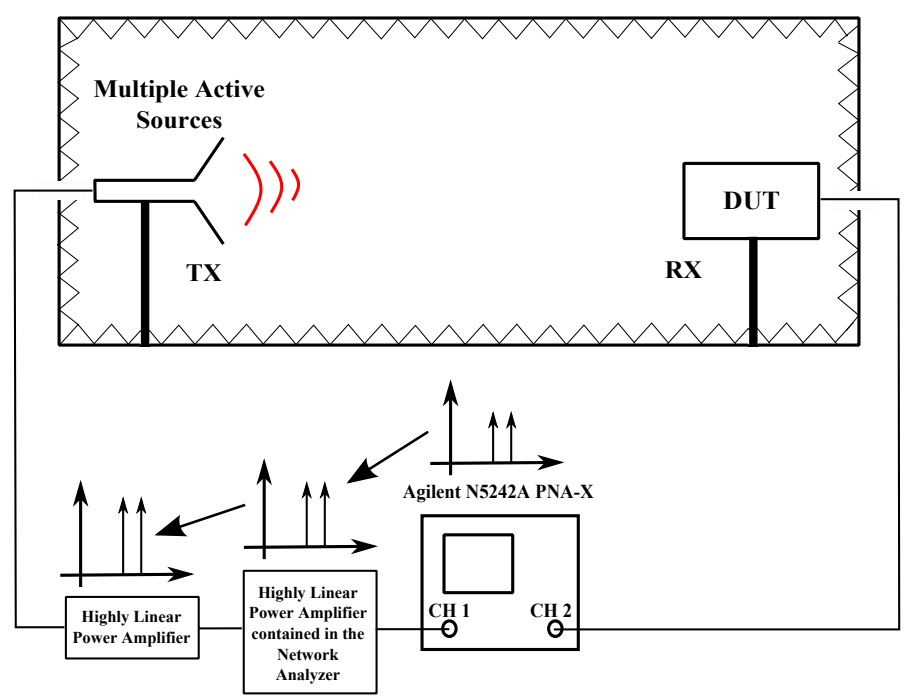

Fig. 5: Anechoic Chamber Setup. 


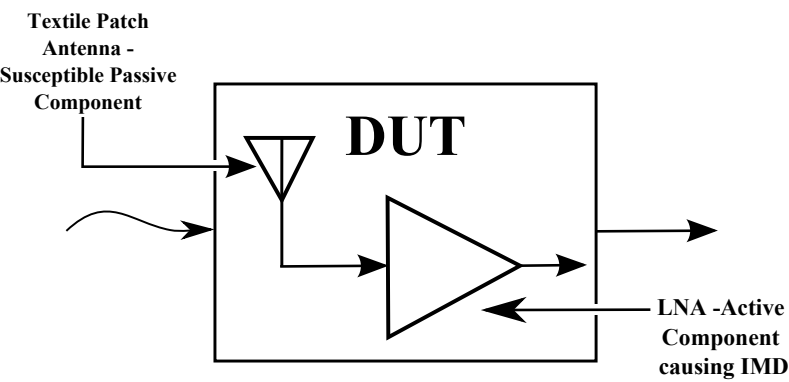

Fig. 6: Passive antenna and LNA. 


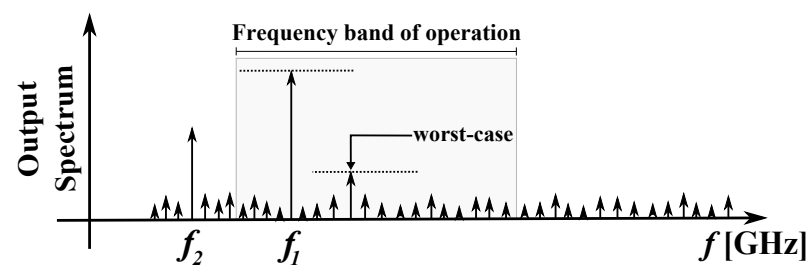

Fig. 7: Worst-case identification for the calculation of the Spurious Free Dynamic Range. 

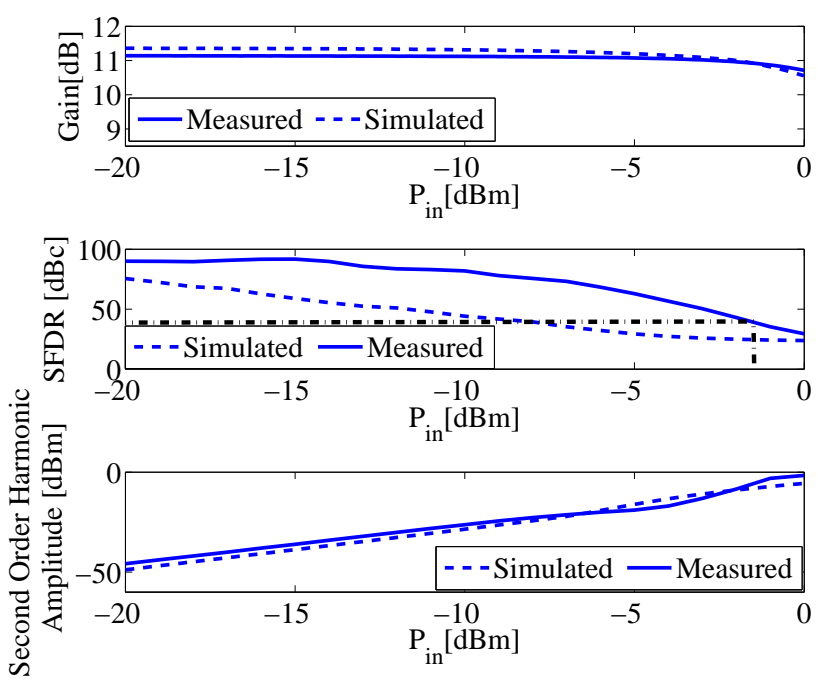

Fig. 8: Gain at $f_{0}=2.45 \mathrm{GHz}$, SFDR for $f_{1}=2.445 \mathrm{GHz}$ and $f_{2}=2.455 \mathrm{GHz}$, and second-order harmonic power for $f_{0}=1.225 \mathrm{GHz}$, simulated and measured. 

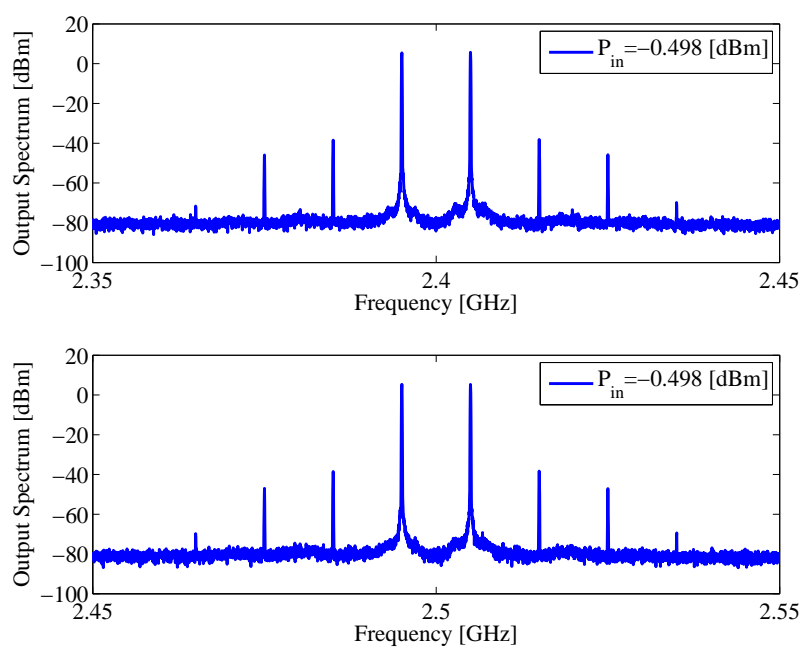

Fig. 9: Output spectrum with $P_{i n}=P_{1 d B}$ at the lower and at the upper side of the considered frequency band, for $\Delta f=10$ $\mathrm{MHz}$ and excitation by two equal power tones $f_{1}$ and $f_{2}$. 


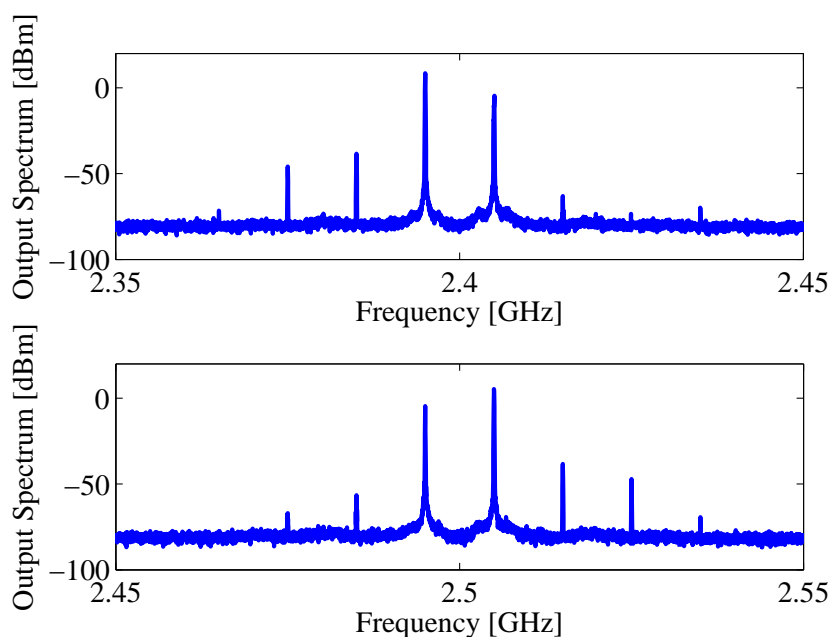

Fig. 10: Measurement results as in Fig. 9 but now for two tones with a power difference of $10 \mathrm{dBm}$. 


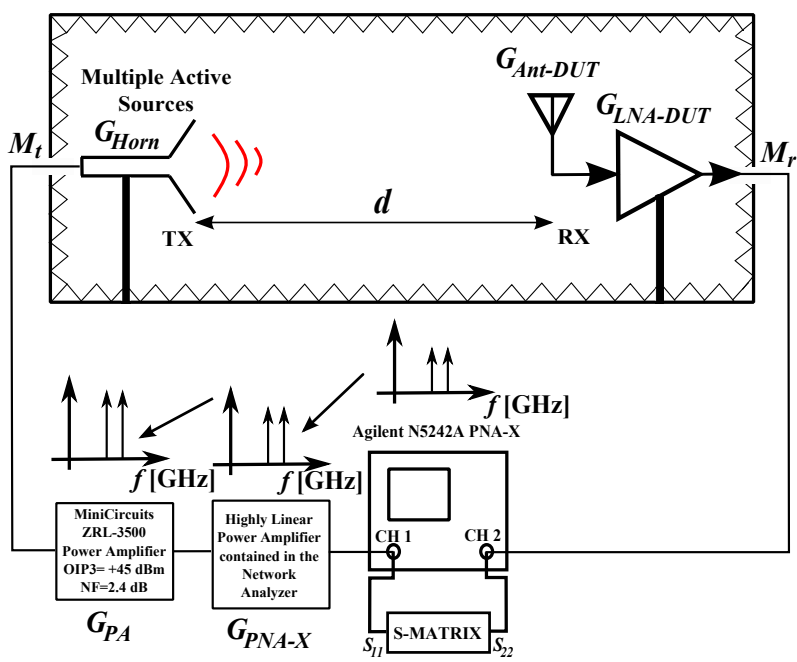

Fig. 11: DUT measurement setup in the anechoic chamber. 

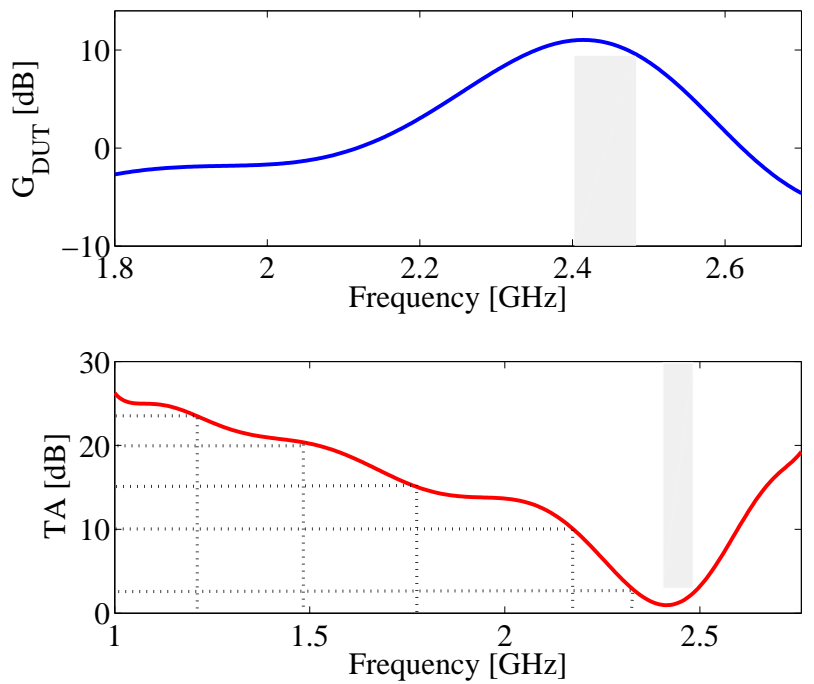

Fig. 12: $G_{D U T}$ and $T A$ as a function of frequency, with $d=10 \lambda_{1}$. 


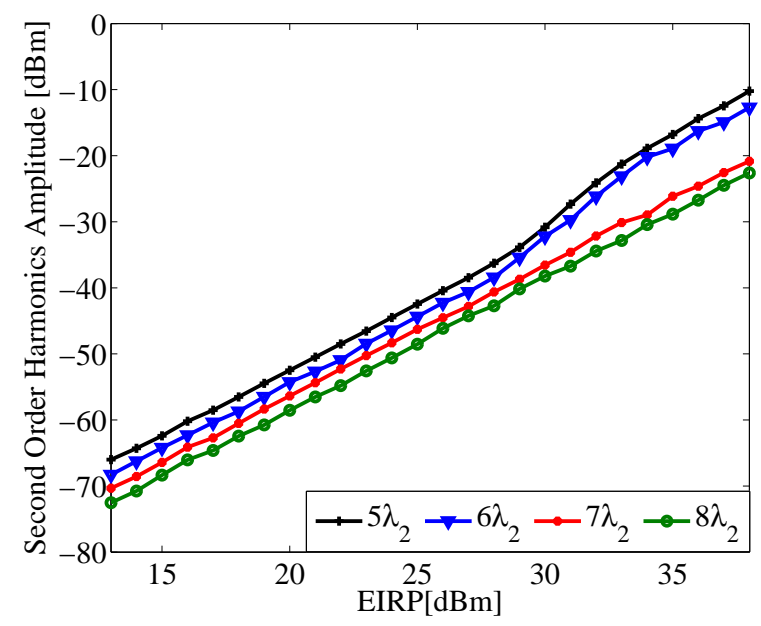

Fig. 13: Second harmonic's power versus distance $d$ between the standard horn and the DUT. 

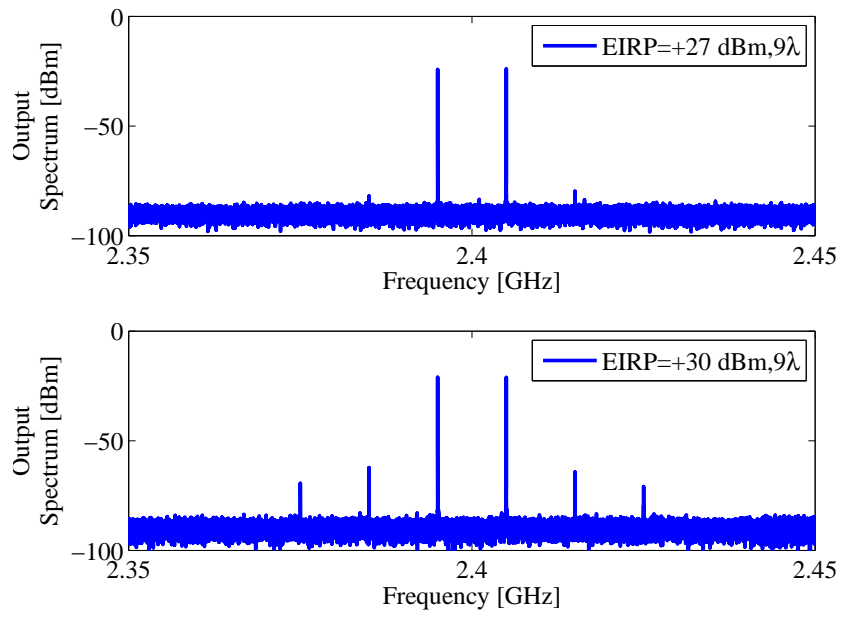

Fig. 14: Output spectrum for the excitation by two equal power tones in the proximity of the lower side of the frequency band with $\mathrm{EIRP}=+27 \mathrm{dBm}$ and $+30 \mathrm{dBm}$ respectively, using $d=9 \lambda_{1}$. 

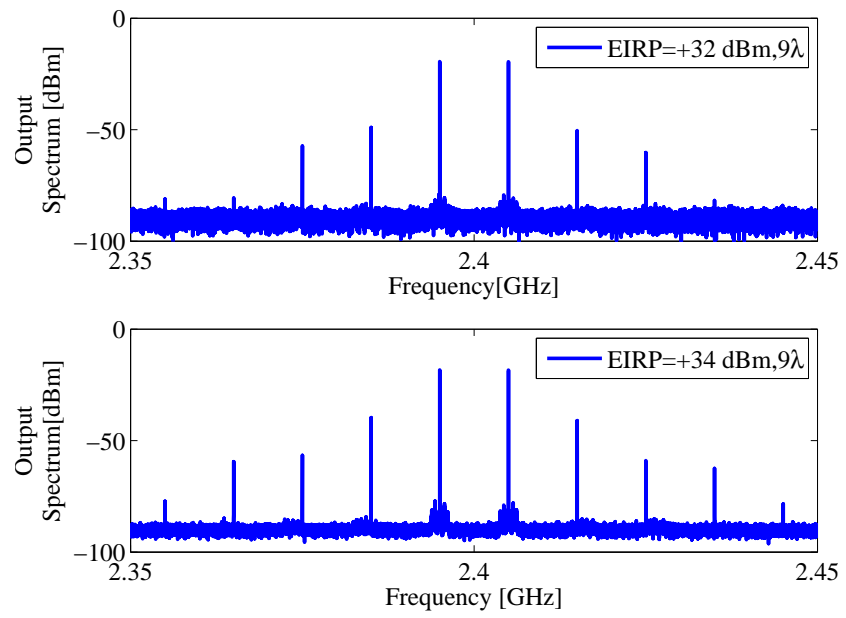

Fig. 15: Output spectrum for the excitation by two equal power tones in the proximity of the lower side of the frequency band with $\mathrm{EIRP}=+32 \mathrm{dBm}$ and $+34 \mathrm{dBm}$ respectively, using $d=9 \lambda_{1}$. 


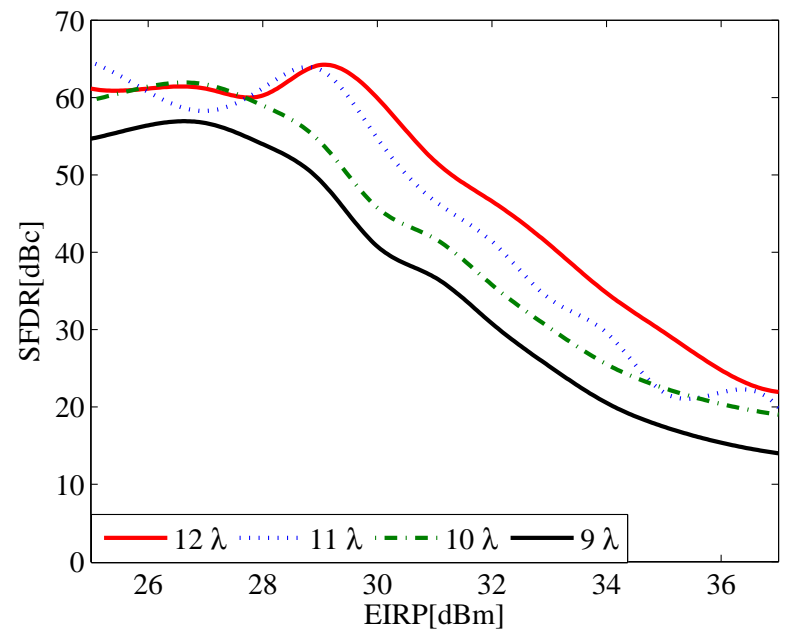

Fig. 16: SFDR as a function of EIRP for two equal power tones and varying distances $\left(\lambda=\lambda_{1}\right)$. 

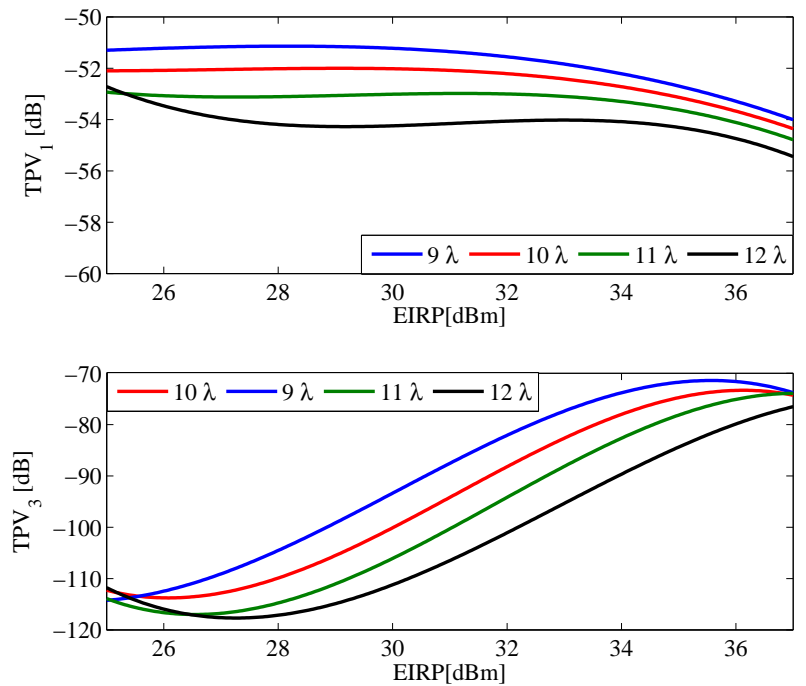

Fig. 17: $T P V_{1}$ and $T P V_{3}$ as a function of EIRP and for variable distances $\lambda=\lambda_{1}$ between horn and DUT. 

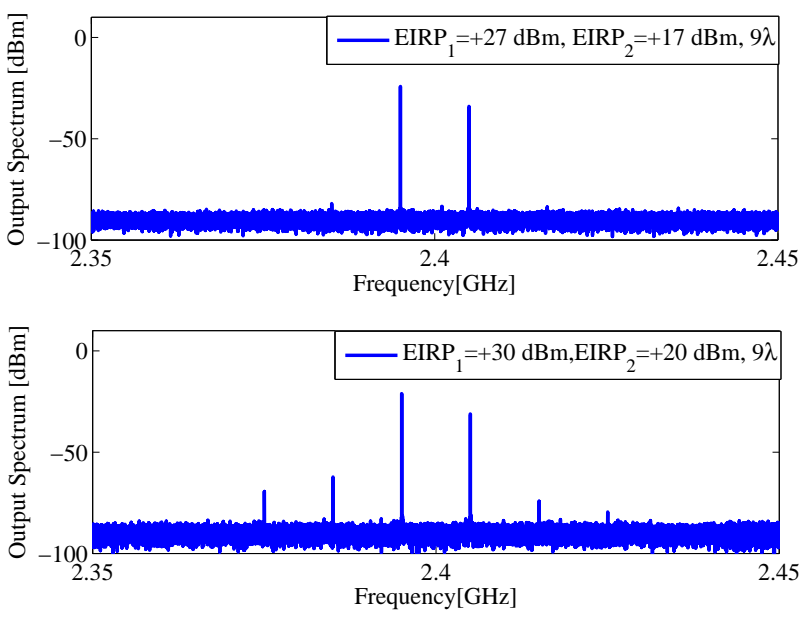

Fig. 18: Output spectrum for the excitation by two tones with different power in the proximity of the lower side of the frequency band and resp. for $E I R P_{1}=27 \mathrm{dBm}, E I R P_{2}=17 \mathrm{dBm}$ and for $E I R P_{1}=30 \mathrm{dBm}, E I R P_{2}=20 \mathrm{dBm}$. 

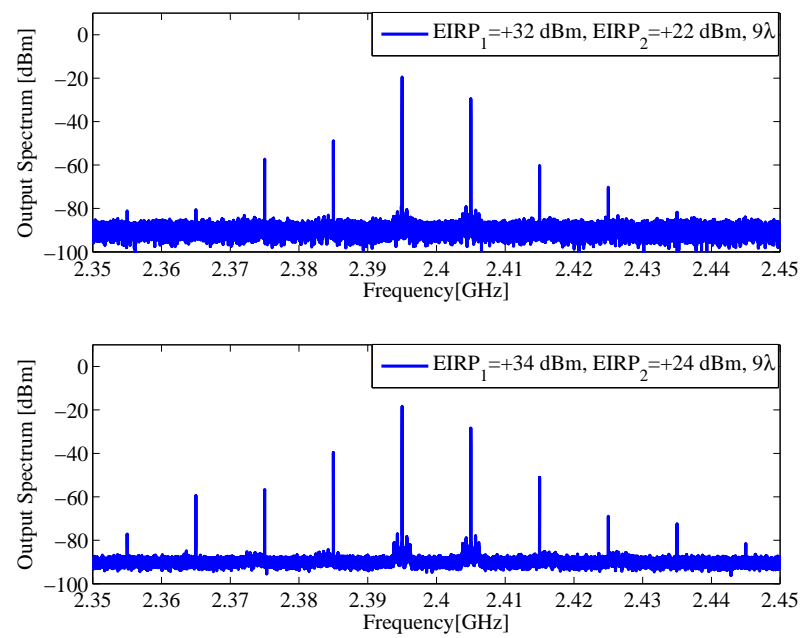

Fig. 19: Output spectrum for the excitation by two tones with different power in the proximity of the lower side of the frequency band and resp. for $E I R P_{1}=32 \mathrm{dBm}, E I R P_{2}=22 \mathrm{dBm}$ and for $E I R P_{1}=34 \mathrm{dBm}, E I R P_{2}=24 \mathrm{dBm}$. 


\section{LIST OF TABLES}

I Main tones power, OIP3, OIP2 measured and simulated. . . . . . . . . . . . . . . . . . . 29

II Link Budget at $2.45 \mathrm{GHz} \ldots \ldots \ldots \ldots$

III Largest in-the-band main tone $P_{\text {Tone }, 1}$ and third-order intermodulation product $P_{\text {Tone }{ }_{3}}$ for varying EIRP values, at a distance $d=9 \lambda_{1} \ldots \ldots \ldots \ldots \ldots$ 
TABLE I: Main tones power, OIP3, OIP2 measured and simulated.

\begin{tabular}{|c|c|c|}
\hline Value & Simulated [dBm] & Measured [dBm] \\
\hline$P_{1 d B} @ f_{0}=2.45 \mathrm{GHz}$ & -0.601 & -0.498 \\
\hline OIP3@ $f_{1}=2.445 \mathrm{GHz}$, & +27.76 & +25.526 \\
$f_{2}=2.455 \mathrm{GHz}$ & & \\
\hline OIP2@ $f_{0}=1.225 \mathrm{GHz}$ & +72.394 & +61.763 \\
\hline
\end{tabular}


TABLE II: Link Budget at $2.45 \mathrm{GHz}$

\begin{tabular}{|c|c|c|c|}
\hline$P_{\text {in }[P N A-X]}$ & $G_{[P A]}$ & $G_{[H O R N]}$ & $M_{t}[\mathrm{~dB}]$ \\
\hline$-20: 1: 0[\mathrm{dBm}]$ & $21[\mathrm{~dB}]$ & $15.35[\mathrm{dBi}]$ & -0.1016 \\
\hline$L_{[\text {cables }]}$ & $G_{[L N A]}$ & $\left.G_{[\text {antDUT }}\right]$ & $M_{r}[\mathrm{~dB}]$ \\
\hline$-0.06[\mathrm{~dB}]$ & $+11[\mathrm{~dB}]$ & $+3.5[\mathrm{dBi}]$ & -0.2174 \\
\hline
\end{tabular}


TABLE III: Largest in-the-band main tone $P_{\text {Tone }, 1}$ and third-order intermodulation product $P_{\text {Tone }, 3}$ for varying EIRP values, at a distance $d=9 \lambda_{1}$.

\begin{tabular}{|c|c|c|c|}
\hline$E I R P[\mathrm{dBm}]$ & $P_{\text {Tone }, 1}[\mathrm{dBm}]$ & $P_{\text {Tone }, 3}[\mathrm{dBm}]$ & SFDR $[\mathrm{dB}]$ \\
\hline+20 & -27.3 & Noise floor & 62.7 \\
\hline+27 & -24.2 & -79.8 & 55.6 \\
\hline+30 & -21.2 & -67.2 & 46.0 \\
\hline+32 & -19.6 & -49.0 & 39.4 \\
\hline+34 & -18.3 & -39.7 & 21.4 \\
\hline
\end{tabular}

